\title{
Aplicación de la electrocoagulación en la eliminación de los metales pesados en los efluentes galvánicos
}

\author{
Application of electrocoagulation in the removal of heavy metals in galvanic \\ effluents
}

\author{
Ernesto León Vilela', Vidal Aramburú Rojas²
}

Recibido: 06/03/2021 - Aprobado: 11/08/2021 - Publicado: 23/12/2021

\begin{abstract}
RESUMEN
La búsqueda de nuevas tecnologías orientadas al tratamiento de los efluentes industriales ha hecho que la electrocoagulación sea considerada como la tecnología emergente con mayor aceptación para el tratamiento de los efluentes galvánicos que por su naturaleza misma contienen altas concentraciones de metales pesados. El objetivo de esta investigación es la eliminación efectiva de la carga final de metales pesados mediante la electrocoagulación en aguas residuales de origen galvánico, utilizando electrodos de aluminio (ánodo) y hierro (cátodo), en un reactor de tipo continúo. Se estudiaron los efectos de parámetros como el pH, densidad de corriente y voltaje, sobre las eficiencias de remoción de los metales pesados. Los resultados experimentales mostraron que el pH óptimo donde se alcanzaron los mayores porcentajes de remoción fue de 6 (Cromo $96.8 \%$, cobre $98.1 \%$, níquel $96.6 \%$ y zinc $98.1 \%$ ). Se encontró que la eficiencia de remoción aumenta con un aumento del voltaje y de la densidad de corriente, alcanzándose a 7.5 voltios y a $19 \mathrm{~A} / \mathrm{m} 2$ los valores más altos de remoción respectivamente. Se puede concluir, con los resultados experimentales obtenidos, que la electrocoagulación es muy eficaz en la eliminación de los metales pesados en los efluentes galvánico.
\end{abstract}

Palabras claves: Efluentes galvánicos; electrocoagulación; remoción de metales pesados; aguas residuales.

\begin{abstract}
The search for new technologies aimed at treating industrial effluents has made electrocoagulation the most widely accepted emerging technology for treating galvanic effluents, which by their very nature contain high concentrations of heavy metals. The objective of this research is the effective elimination of the final heavy metal load through electrocoagulation in wastewater of galvanic origin, using aluminum (anode) and iron (cathode) electrodes, in a continuous reactor. The effects of parameters such as $\mathrm{pH}$, current density and voltage, on the removal efficiencies of heavy metals were studied. The experimental results showed that the optimum pH where the highest removal percentages were reached was 6 (Chrome $96.8 \%$, copper $98.1 \%$, nickel $96.6 \%$ and zinc $98.1 \%$ ). Removal efficiency was found to increase with an increase in voltage and current density, reaching 7.5 volts and $19 \mathrm{~A} / \mathrm{m} 2$ the highest removal values respectively. It can be concluded, with the experimental results obtained, that electrocoagulation is very effective in removing heavy metals from galvanic effluents.
\end{abstract}

Keywords: Galvanic effluents; electrocoagulation; heavy metal removal; wastewater.

1 Universidad Nacional Mayor de San Marcos, Facultad de Ingeniería Geológica, Minera, Metalúrgica y Geográfica, Unidad de Posgrado, Lima, Perú. Egresado de Maestría.

Autor para correspondencia: ernesto.leon2@unmsm.edu.pe / eleonvi@gmail.com - ORCID: https://orcid.org/0000-0002-9676-5897

2 Universidad Nacional Mayor de San Marcos, Facultad de Ingeniería Geológica, Minera, Metalúrgica y Geográfica, Unidad de Posgrado, Lima, Perú. Profesor Principal.

E-mail: varamburur@unmsm.edu.pe - ORCID: https://orcid.org/0000-0001-7411-3866 


\section{INTRODUCCIÓN}

Los procesos de tratamientos de superficies tienen una gran importancia en todas las esferas de la economía y la sociedad, ya que estos garantizan recubrimientos protectores anticorrosivos con una alta durabilidad, además de propiciar una estética agradable (Chávez Porras et al., 2009). Esta actividad es una de las principales industrias causantes de contaminación a través de descargas de agua de enjuague, las cuales contienen substanciales cantidades de metales tóxicos, tales como zinc, cobre, níquel, cromo y cadmio (Ardila Padilla, 2005).

Ante tales desafíos comenzó la preocupación por dar solución a la problemática ambiental, sobre todo dando mucha importancia al tratamiento de residuales. De esta forma, muchos de los sectores industriales se vieron obligados a implementar medidas que minimicen los impactos ambientales de manera negativa (Restrepo et al., 2006), ya sea a través de la implementación de prácticas de prevención de la contaminación o del tratamiento de aguas residuales antes de ser vertidos al medio ambiente (Chávez Porras et al., 2009, Acosta Niño et al., 2013). Es así como la electrocoagulación, como proceso que emplea los fundamentos de la electroquímica, la coagulación y flotación o precipitación, puede emplearse para eliminar los contaminantes del agua (Acosta Niño et al., 2013). La descontaminación electroquímica es el resultado de la combinación de varios procesos, que pueden resumirse en: electroreducción, electrooxidación, electrocoagulación y electroflotación (Lavorante et al., 2011).

La utilización de este tipo de tecnología implica utilizar un reactor de electrocoagulación (celda electrolítica) cuyos conductores de corriente (electrodos), son sumergidos en el fluido a tratar, el que contiene los metales o sustancias que van a ser removidos y que es aprovechado como medio electrolítico (Acosta Niño et al., 2013). Mollah et al. (2004), sostiene que la electrocoagulación es un proceso simple y muy eficiente donde se genera el agente de floculación por electro oxidación del ánodo de sacrificio, mayormente hecho de hierro o aluminio. En el proceso de electrocoagulación, generalmente el tratamiento se realiza sin añadir ningún agente químico coagulante o floculante, con el propósito de reducir la cantidad de lodos que se elimina (Acosta Niño et al., 2013).

En este proceso, la generación de iones metálicos tiene lugar en el ánodo, debido a la oxidación del electrodo. En el cátodo observamos que se produce hidrógeno debido a la hidrólisis del agua. Las reacciones generales son las siguientes:

En el ánodo:

$$
\begin{aligned}
& \mathrm{Fe} \rightarrow \mathrm{Fe}^{2+}+2 \mathrm{e}^{-} \_(1) \\
& \mathrm{Al} \rightarrow \mathrm{Al}^{3+}+3 \mathrm{e}^{-}-(3) \\
& \mathrm{Zn} \rightarrow \mathrm{Zn}^{2+}+2 \mathrm{e}-2 \mathrm{Cr} \\
& \mathrm{Cr}_{2} \mathrm{O}_{7}{ }^{2+}+6 e^{-+}+7 \mathrm{H}_{2} \mathrm{O} \rightarrow 2 \mathrm{OH}^{-} \\
& \mathrm{Cr}^{6+}+3 \mathrm{Fe}^{2+} \rightarrow \mathrm{Cr}^{3+}+3 \mathrm{Fe}^{3+}
\end{aligned}
$$

$$
\begin{aligned}
& \mathrm{Cu} \rightarrow \mathrm{Cu}^{2+}+2 \mathrm{e} \\
& \mathrm{Ni} \rightarrow \mathrm{Ni}^{2+}+2 \mathrm{e}^{-}
\end{aligned}
$$

Finalmente, estos metales forman hidróxidos metálicos como sigue:

$\mathrm{Me}^{n+}+n \mathrm{OH}^{-} \rightarrow \mathrm{Me}(\mathrm{OH})_{n(s)}$

En el cátodo:

$$
2 \mathrm{H}_{2} \mathrm{O}+2 \mathrm{e}^{-} \rightarrow \mathrm{H}_{2(\mathrm{~g})}+2 \mathrm{OH}^{-}
$$

Como nos muestran las reacciones anteriores, en el ánodo de aluminio, se desarrollan dos reacciones de separación. Primero, el ánodo se disuelve produciendo en forma continua hidróxidos poliméricos metálicos que son muy buenos agentes coagulantes. Asimismo, se da la electrólisis del agua en una reacción paralela produciendo pequeñas burbujas de oxígeno en el ánodo y de hidrógeno en el cátodo (Franco Cogollo \& Meza Cárcamo, 2017). Los ánodos metálicos consumibles se utilizan continuamente para producir cationes metálicos polivalentes en la zona del ánodo. Estos cationes neutralizan la carga negativa de las partículas en movimiento hacia los ánodos por la producción de cationes polivalentes a partir de la oxidación del ánodo de sacrificio ( $\mathrm{Fe} \mathrm{o} \mathrm{Al}$ ) y los gases de electrólisis como el hidrógeno desprendido en el ánodo y el oxígeno generado en el cátodo (Satish.I., 2013).

El presente trabajo de investigación se justifica por proponer la tecnología de electrocoagulación como una alternativa viable en la eliminación de los metales pesados en los efluentes galvánicos, evaluando la remoción como parámetro de respuesta en cada caso, relacionando la concentración inicial $\left(\mathrm{CMet}_{\mathrm{i}}\right)$ y final $\left(\mathrm{CMet}_{\mathrm{f}}\right)$ del metal pesado, expresado como el ciento de remoción, por la ecuación que se expresa a continuación:

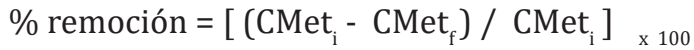

\section{MÉTODOS}

En la presente investigación se evalúo la técnica de electrocoagulación como alternativa para el tratamiento de los efluentes industriales de origen galvánico, con el propósito de reducir las concentraciones finales de los metales pesados, para lo cual se realizó una caracterización inicial y posterior al tratamiento por electrocoagulación, optimizando los parámetros de proceso para determinar su porcentaje de remoción orientado al objetivo planteado.

\subsection{Materiales}

Parael proyecto se construyó una celda de electrocoagulación de tipo continuo en acrílico, cuyas medidas son: $31 \mathrm{~cm}$ de altura, $17 \mathrm{~cm}$ de ancho y $36 \mathrm{~cm}$ de largo, volumen total de la celda de 8.5 litros y un flujo de $1 \mathrm{lt} / \mathrm{min}$. El sistema consta de cuatro compartimentos situados en continuo, haciendo una rampa con un ángulo de 20 grados (Jaramillo Páez et al., 2005). De acuerdo con la figura 1, tenemos: (a) diseño del reactor electrolítico continuo, en (b) se muestra la celda EC en operación continua. 
En el sistema, tres compartimentos están equipados cada uno con 4 electrodos: 2 electrodos de hierro y 2 electrodos de aluminio, en forma alterna, de dimensiones $150 \times 152 \times 2.3 \mathrm{~mm}$ que se compraron a un proveedor local, con una separación entre ellos de $10 \mathrm{~mm}$. Los electrodos están distribuidos en una configuración en forma monopolar en paralelo y conectados a una fuente de alimentación de corriente continua de energía de 0 - 20 Amperios y un rango de $0-40 \mathrm{~V}$ regulable. El $\mathrm{pH}$ de estudio, para las pruebas experimentales, varió en 6,8 a 10, y para su medición se utilizó un Hanna HI8314. El efecto del potencial eléctrico (voltaje) aplicado fue de $6,6.5,7$ y 7.5 voltios, y para su medición se utilizó un multímetro PHYWE.

El efluente industrial galvánico seleccionado para las pruebas de electrocoagulación proviene de las descargas de los procesos industriales de una planta industrial galvánica, que fue monitoreada en la Empresa de Fabricación de Suministros Textiles. Los efluentes tenían una coloración verdosa amarronada turbia y sólidos suspendidos. En la Tabla 1, se muestras las condiciones iniciales:

\subsection{Metodología}

Se trabajó con un volumen de solución madre total de 600 litros de efluente, para todas las corridas incluyendo las réplicas, el cual fue dividido en tres lotes iguales de 200 litros. Para acondicionar los tres niveles de $\mathrm{pH}, 6,8$ y 10, se utilizó ácido sulfúrico $\left(\mathrm{H}_{2} \mathrm{SO}_{4}\right)$ y soda caústica $(\mathrm{NaOH})$. Los electrodos fueron acondicionados con una distribución tipo monopolar en paralelo, como se puede observar en la figura 2, utilizándose electrodos de hierro y aluminio en

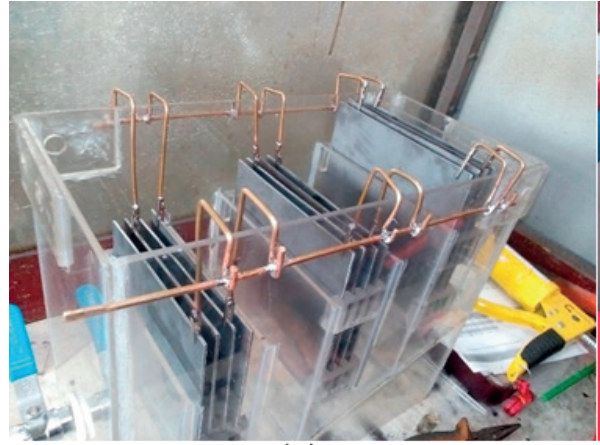

(a)

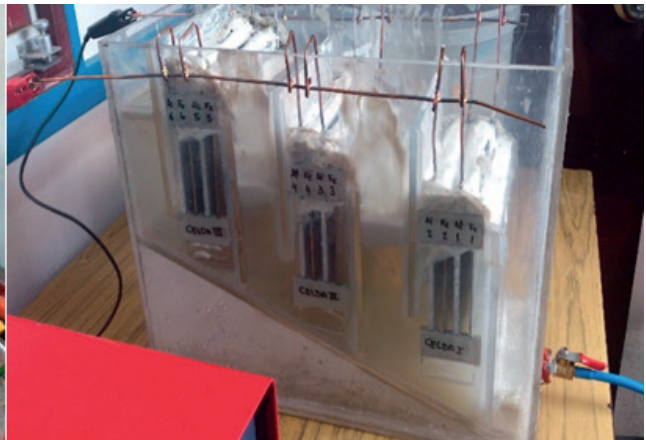

(b)

Figura 1. (a) Diseño de la celda electrolítica continua. (b) Celda EC en operación continua.

Fuente: propia del autor

Tabla 1. Caracterización inicial del efluente industrial galvánico

\begin{tabular}{ccccc}
\hline Descripción de Muestra & Cromo Total $(\mathrm{Cr}) \mathrm{mg} / \mathrm{L}$ & Cobre total $(\mathrm{Cu}) \mathrm{mg} / \mathrm{L}$ & Níquel total $(\mathrm{Ni}) \mathrm{mg} / \mathrm{L}$ & Zinc total $(\mathrm{Zn}) \mathrm{mg} / \mathrm{L}$ \\
\hline Muestra patrón pH 6 & 256.95 & 125.788 & 204.03 & 42.297 \\
Muestra Patrón pH 8 & 92.85 & 301.19 & 182.12 & 193.216 \\
Muestra Patrón pH 10 & 0.84 & 281.55 & 10.39 & 13.084 \\
Temperatura & & & $23^{\circ} \mathrm{C}$ & \\
pH & & 7.01 & \\
turbidez $(\mathrm{FTU})$ & & 996 & \\
conduct. $(\mu \mathrm{S} / \mathrm{cm})$ & & 5.8 & \\
\hline
\end{tabular}

Fuente: Elaboración propia

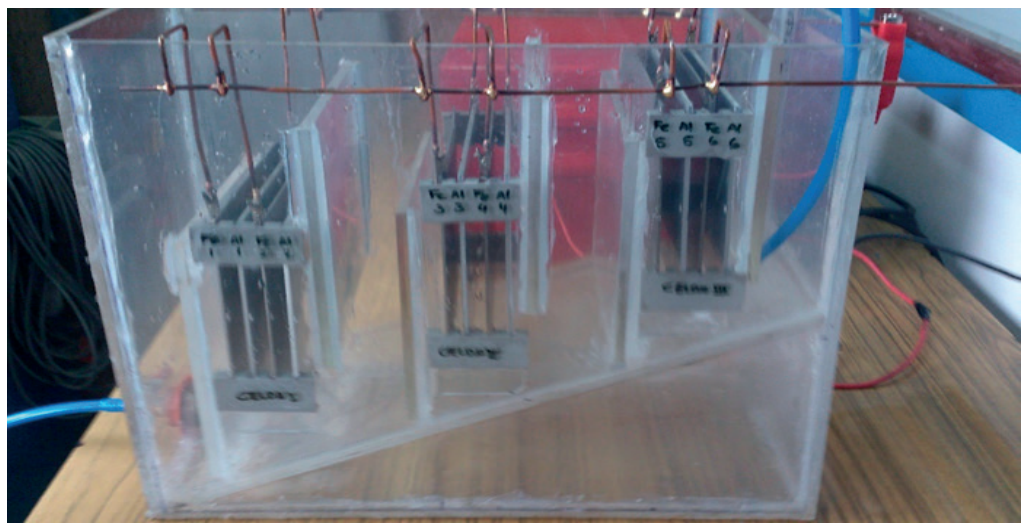

Figura 2. Distribución alterna de electrodos de aluminio y hierro en cada compartimiento. Fuente: Elaboración propia 
forma alternada por cada compartimiento (celda) y una distancia entre ellos de $10 \mathrm{~mm}$.

Cada lote de 200 litros de efluente, con el pH acondicionado, se subdividió a 50 litros de acuerdo con los cuatro niveles establecidos para el factor voltaje (25 litros para prueba y 25 litros para réplica). Las muestras experimentales, se recolectan recogiéndose luego de 20 minutos de proceso continuo, distancia entre los electrodos fue de $10 \mathrm{~mm}$ con un área total efectiva de $4195 \mathrm{~cm}^{2}$. Todos los parámetros del proceso de electrocoagulación que se utilizaron se muestran en la tabla 2. También al final de cada etapa del experimento se determinó el peso del lodo producido.

Tabla 2. Parámetros del proceso de Electrocoagulación

\begin{tabular}{cc}
\hline Parámetros & Valores \\
\hline Tipo de muestra & Agua residual industrial (Efluente galvánico) \\
Tipo de operación & Continuo \\
Volumen de celda electrolítica & $2.8 \mathrm{~L}$ \\
Tiempo de sedimentación & 60 min \\
Material de electrodos & Hierro $(06$ und.) y aluminio (06 und.) \\
Configuración de electrodos & Monopolar en paralelo \\
Espacio entre electrodos & $10 \mathrm{~mm}^{2}$ \\
Área de electrodos & $5472\left(\mathrm{~cm}^{2}\right)$ \\
Área efectiva de electrodos & $4195\left(\mathrm{~cm}^{2}\right)$ \\
Número de ensayos & 24 ensayos $(12$ de pruebas y 12 de réplica) \\
Niveles de pH & 6,8 y 10 \\
Niveles de voltaje & $6.0,6.5,7.0$ y 7.5 voltios \\
Temperatura de proceso & $23^{\circ} \mathrm{C}$ \\
\hline Fuente: propia del autor
\end{tabular}

Fuente: propia del autor

Previo de cada corrida, los electrodos fueron debidamente lijados y decapados en solución de ácido clorhídrico $(\mathrm{HCl})$ al $0.1 \%$ en peso y posteriormente enjuagados con agua destilada después de cada corrida. La eficiencia de remoción, de cada prueba, se evalúo conociendo las concentraciones iniciales y finales de cada prueba. Todas las muestras fueron filtradas con papel filtro Whatman grado 40, antes de ser enviadas a un laboratorio certificado. Luego de las corridas de electrocoagulación, todas las muestras fueron enviadas al laboratorio certificado para su respectivo análisis de los parámetros que permitan identificar y cuantificar la influencia de las variables en estudio ( $\mathrm{pH}$, densidad de corriente y el voltaje) en la eficiencia de remoción de los metales pesados.

El procesamiento de los datos experimentales, luego de aplicar el diseño experimental, fue mediante el software Minitab ${ }^{\circledR}$ versión 20 para la revisión estadística, así como algunas herramientas de $\operatorname{Excel}^{\circledR}$ para la elaboración de tablas.

\section{RESULTADOS}

El proceso de electrocoagulación puede verse afectado por varios parámetros operativos, como el potencial eléctrico (voltaje), densidad de corriente y el $\mathrm{pH}$ inicial. En el presente estudio, se evaluaron los diferentes parámetros y sus efectos en la remoción del cromo, cobre, níquel y zinc de las aguas residuales de origen galvánico utilizando la técnica de la electrocoagulación. Los resultados promedios se muestran en la tabla 3, para cada muestra enviada.

Tabla 3. Porcentaje de remoción (\%) de metales pesados en efluentes tratados

\begin{tabular}{cccccccc}
\hline Muestra & pH & $\begin{array}{c}\text { Voltaje } \\
\text { (voltios) }\end{array}$ & $\begin{array}{c}\text { dens. } \\
\text { corriente } \\
\text { (Amp/m2) }\end{array}$ & $\begin{array}{c}\text { remoc. } \\
\text { Cr }\end{array}$ & $\begin{array}{c}\text { remoc. } \\
\text { Cu }\end{array}$ & $\begin{array}{c}\text { remoc. } \\
\text { Ni }\end{array}$ & $\begin{array}{c}\text { remoc. } \\
\text { Zn }\end{array}$ \\
\hline M1 & 6 & 6 & 13 & $89.8 \%$ & $92.5 \%$ & $90.7 \%$ & $86.8 \%$ \\
M2 & 6 & 6.5 & 15 & $94.0 \%$ & $94.6 \%$ & $94.8 \%$ & $92.6 \%$ \\
M3 & 6 & 7 & 17 & $96.2 \%$ & $96.4 \%$ & $95.3 \%$ & $96.5 \%$ \\
M4 & 6 & 7.5 & 19 & $96.8 \%$ & $98.1 \%$ & $96.6 \%$ & $98.1 \%$ \\
M5 & 8 & 6 & 13 & $71.0 \%$ & $89.4 \%$ & $81.5 \%$ & $84.7 \%$ \\
M6 & 8 & 6.5 & 15 & $88.3 \%$ & $93.1 \%$ & $91.7 \%$ & $92.8 \%$ \\
M7 & 8 & 7 & 17 & $89.9 \%$ & $93.6 \%$ & $95.5 \%$ & $94.6 \%$ \\
M8 & 8 & 7.5 & 19 & $93.6 \%$ & $96.7 \%$ & $96.3 \%$ & $96.4 \%$ \\
M9 & 10 & 6 & 13 & $38.1 \%$ & $80.1 \%$ & $45.4 \%$ & $49.8 \%$ \\
M10 & 10 & 6.5 & 15 & $47.6 \%$ & $81.4 \%$ & $62.4 \%$ & $55.3 \%$ \\
M11 & 10 & 7 & 17 & $64.3 \%$ & $84.0 \%$ & $73.5 \%$ & $61.8 \%$ \\
M12 & 10 & 7.5 & 19 & $71.4 \%$ & $87.6 \%$ & $79.3 \%$ & $70.7 \%$ \\
\hline Fuente: Resultados obtenidos por el laboratorio certificado AGQ & & & &
\end{tabular}

Fuente: Resultados obtenidos por el laboratorio certificado AGQ

Como se observa en la Tabla 3, la muestra M4 es la que obtuvo los mayores porcentajes de remoción. Estos valores promedios obtenidos, con la remoción porcentual producida, se encuentran dentro de los rangos que la normativa ambiental exige (D.S. $\mathrm{N}^{\circ}$ 021-2009VIVIENDA).

\subsection{Efecto del pH inicial sobre la eficiencia de remoción de metales}

En estudios previos (Chen et al., 2000) se estableció que el $\mathrm{pH}$ tiene un efecto considerable sobre la eficiencia del proceso de electrocoagulación. De acuerdo con los datos experimentales, observamos en la figura 3 el efecto del $\mathrm{pH}$ inicial, sobre la eficiencia de remoción de los metales pesados, que alcanzó los mayores valores a un $\mathrm{pH}$ 6, obteniéndose $96.8 \%, 98.1 \%, 96.6 \%$ y $98.1 \%$, para el $\mathrm{Cr}, \mathrm{Cu}, \mathrm{Ni}$ y $\mathrm{Zn}$ respectivamente cuando se utiliza como electrodos Hierro (cátodo) y aluminio (ánodo). Entre el rango de $\mathrm{pH} 6$ a 8 los porcentajes de reducción del $\mathrm{Cu}$, Ni y $\mathrm{Zn}$, permanecen por encima del $80 \%$ a excepción del Cr y luego todos disminuyen a $\mathrm{pH} 10$, siendo el $\mathrm{Cr}$ el que alcanza el menor valor (38.1\%). La disminución de la eficiencia de remoción a un $\mathrm{pH}$ alcalino fue descrita por Adhoum et al. (2004) que le atribuye a un comportamiento anfótero del $\mathrm{Al}(\mathrm{OH})_{3}$ que conduce a aniones monoméricos $\mathrm{Al}(\mathrm{OH})_{4}^{-}$.

Durante el proceso se observó aumento del pH para el valor de pH 6, lo que según Vik et al. (1984) se atribuye al desprendimiento de hidrógeno y a la generación de iones $\mathrm{OH}^{-}$en el cátodo. Sin embargo, esto fue impugnado por Chen et al. (2000), quienes explicaron este aumento por la liberación de $\mathrm{CO}_{2}$ de las aguas residuales debido a la perturbación de las burbujas de $\mathrm{H}_{2}$. De hecho, a un 
pH bajo, el $\mathrm{CO}_{2}$ está saturado en exceso en las aguas residuales y puede liberarse durante el desprendimiento de $\mathrm{H}_{2}$, provocando un aumento del $\mathrm{pH}$. A valores mayores de 8 de $\mathrm{pH}$ (medio alcalino), no se observa aumento, pero si una ligera disminución de $\mathrm{pH}$. De acuerdo con la literatura (Konstantinos et al., 2011) este comportamiento en medio alcalino se debe al consumo de los iones $\mathrm{OH}^{-}$, generados en el cátodo, por los iones de $\mathrm{Al}^{+3}$ generado en el ánodo formando flóculos de $\mathrm{Al}(\mathrm{OH})_{3}$. Además, los iones $\mathrm{OH}^{-}$se combinan parcialmente con los iones $\mathrm{Ni}^{+2}, \mathrm{Cu}^{+2}, \mathrm{Zn}^{+2}$ y $\mathrm{Cr}^{+6}$ para formar hidróxidos insolubles que precipitan, por esta razón es que en medio alcalino no se registra aumento de $\mathrm{pH}$.

\subsection{Efecto del voltaje en la eficiencia de remoción de metales}

El voltaje aplicado es uno de los parámetros operativos que directamente afecta a la eficiencia de remoción de los metales.

Las pruebas se realizaron a $6,6.5,7$ y 7.5 voltios. Los valores máximos de los ensayos se obtuvieron a $7.5 \mathrm{~V}$ con $96.8 \%, 98.1 \%, 96.6 \%$ y $98.1 \%$ para el $\mathrm{Cr}, \mathrm{Cu}, \mathrm{Ni}$ y $\mathrm{Zn}$ respectivamente. Al aumentar el voltaje, como observamos en la figura 4, el porcentaje de remoción aumenta significativamente.

Estos resultados sugieren $7.5 \mathrm{~V}$ como potencial eléctrico (voltaje) óptimo para el tratamiento de efluentes que contengan Cr, Cu, Ni y Zn. Según Golder et al. (2007), también observó el mismo comportamiento al eliminar metales $(\mathrm{Cu}, \mathrm{Cr}$ y Ni) y lo atribuyeron al aumento de la tasa de generación de burbujas, influyendo en la transferencia de masa en los electrodos, lo que resulta en una mayor cantidad de precipitado para la eliminación de contaminantes.

Según los trabajos hechos por Nouri et al. (2010) en la remoción del $\mathrm{Cu}$ y Zn de aguas sintéticas, después de 15 a 60 minutos. cuanto mayor sea el voltaje aplicado al sistema, en el rango entre 20 a $40 \mathrm{~V}$, te tendrán eliminaciones más efectivas, las cuales estaban asociadas a las concentraciones iniciales, $\mathrm{pH}$, voltaje y tiempo de contacto. Observó que al aumentar el potencial eléctrico (voltaje) se consumirán

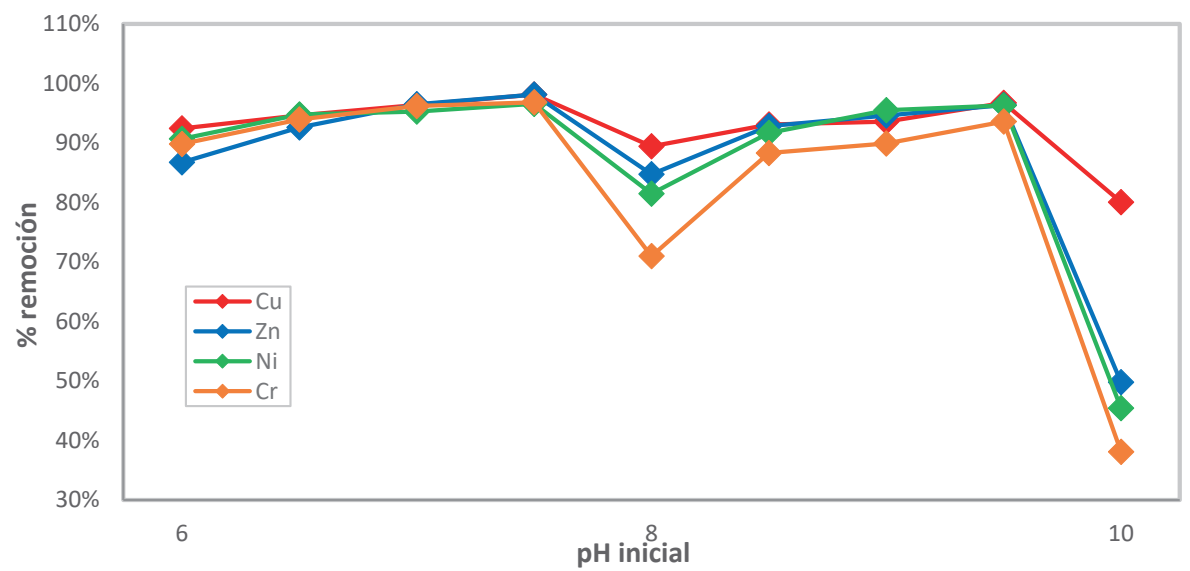

Figura 3. Efecto del pH inicial en la remoción de metales utilizando electrodo ánodo (aluminio) y cátodo (hierro). Condiciones: voltaje $7.5 \mathrm{~V}$, densidad de corriente $19 \mathrm{~A} / \mathrm{m}^{2}$, distancia entre electrodos $10 \mathrm{~mm}, \mathrm{pH}$ 6,8 y 10 , proceso continuo.

Fuente: Elaboración propia

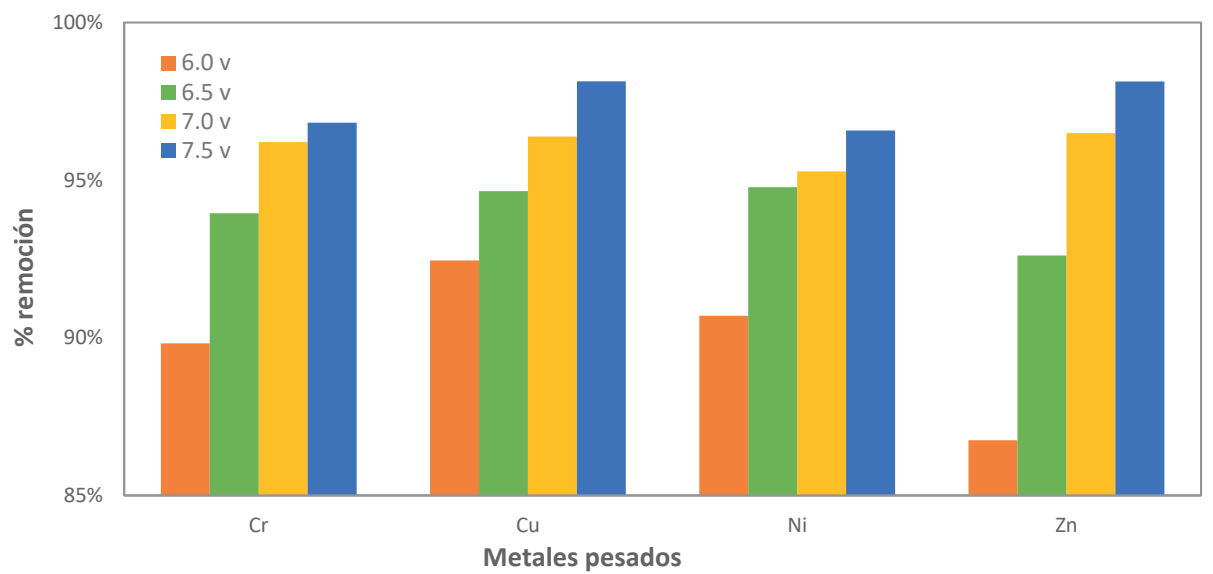

Figura 4. Efecto del voltaje sobre la eficiencia de remoción. Condiciones: $\mathrm{pH} 6$, distancia entre electrodos $10 \mathrm{~mm}$, proceso continuo, voltajes $6,6.5,7$ y $7.5 \mathrm{~V}$.

Fuente: Elaboración propia 
más electrodos en el proceso. También, cuanto mayor sea las concentraciones de los contaminantes en la solución, mayor será el consumo del electrodo. En nuestro estudio, los resultados obtenidos respecto al consumo del electrodo (Al-ánodo) a diferentes tensiones, se muestran en la tabla 4 , en donde para un proceso continuo de 50 minutos, el consumo del aluminio como ánodo a 7.5 voltios es 28 gramos en comparación con el realizado a 6 voltios que consume 2.9 gramos, representando la relación entre el consumo y el peso inicial de $1.30 \%$ y $0.10 \%$ para 7.5 y 6 voltios respectivamente.

Tabla 4. Efecto del voltaje en el consumo de electrodo (aluminio ánodo). Condiciones: $\mathrm{pH}$ 6, distancia entre electrodos $10 \mathrm{~mm}$, proceso continuo, tensión $6,6.5,7$ y 7.5 voltios

\begin{tabular}{lcccc}
\hline Al - ánodo & $6 \mathrm{v}$ & $6.5 \mathrm{v}$ & $7 \mathrm{v}$ & $7.5 \mathrm{v}$ \\
\hline Consumo total, gr & 2.9 & 7.8 & 19 & 28 \\
gr consumidos / gr peso inicial (\%) & $0.10 \%$ & $0.40 \%$ & $0.90 \%$ & $1.30 \%$ \\
\hline
\end{tabular}

Fuente: propia del autor

\subsection{Efecto de la densidad de corriente en la eficiencia de remoción de metales}

De acuerdo con los resultados obtenidos, la densidad de corriente tiene un impacto significativo en la eficiencia de remoción de metales pesados. Para investigar el efecto, se realizaron experimentos a diferentes densidades de corrientes que variaron entre 13 y $19 \mathrm{~A} / \mathrm{m} 2$. La tasa de dosificación de coagulante no solo lo determina la densidad de corriente, sino también es responsable de la tasa y tamaño de producción de burbujas y del crecimiento de flóculos (Kobya et al., 2003), que puede influir en la eficacia del tratamiento de la electrocoagulación. Un aumento de la densidad de corriente aumenta la densidad de burbujas y su tamaño disminuye (Khosla et al., 1991), lo que resulta en un mayor flujo hacia arriba y una remoción más rápida y flotación de lodos.

Podemos observar en la figura 5 , el efecto de la densidad de corriente en la eficiencia de remoción de los metales en estudio, que aumenta conforme se va incrementando la densidad de corriente. Los valores de remoción más alta se obtuvieron a una densidad de corriente de $19 \mathrm{~A} / \mathrm{m} 2$ con eficiencias de $96.8 \%, 98.1 \%, 96.6 \%$ y $98.1 \%$ para el $\mathrm{Cr}$, $\mathrm{Cu}, \mathrm{Ni}$ y $\mathrm{Zn}$ respectivamente. De acuerdo con Holt et al. (2002), este comportamiento se puede atribuir al hecho que, a alta corriente, la cantidad de iones oxidados del ánodo se incrementa dando como resultado una mayor cantidad de precipitado, removiendo los contaminantes en forma más eficiente y rápida.

\section{CONCLUSIÓN}

Se investigó la técnica de electrocoagulación utilizando electrodos de aluminio (ánodo) y hierro (cátodo), mediante los efectos de los diferentes parámetros de proceso como el $\mathrm{pH}$ inicial, la densidad de corriente y el voltaje en la remoción de metales pesados de los efluentes galvánicos.

El proceso de electrocoagulación fue aplicado con éxito al tratamiento de agua residual de origen galvánico, encontrándose que la eficiencia de remoción depende del $\mathrm{pH}$, de la densidad de corriente y del voltaje aplicado.

La eficiencia de remoción más efectiva de los metales pesados como el cromo, cobre, níquel y zinc se obtiene con un voltaje de 7.5 voltios, $\mathrm{pH} 6$ y una densidad de corriente de $19 \mathrm{~A} / \mathrm{m}^{2}$, siendo las condiciones óptimas del proceso que se aplicó simultáneamente para los cuatro metales pesados, alcanzándose las eficiencias máximas de 96.8\%, 98.1\%, $96.6 \%$ y $98.1 \%$ para el $\mathrm{Cr}, \mathrm{Cu}$, Ni y Zn, respectivamente.

Los resultados mostraron que la eficiencia de remoción de metales pesados aumenta con el incremento de la densidad de corriente y el voltaje. La eficiencia de remoción también aumenta con la disminución del $\mathrm{pH}$ inicial, encontrándose que en el rango de $\mathrm{pH}$ de 6 a 8 , las eficiencias de remoción del cobre, níquel y zinc se eliminaron más rápidamente que el cromo hexavalente.

La electrocoagulación es un método seguro, confiable y eficiente para eliminar los metales pesados de las aguas

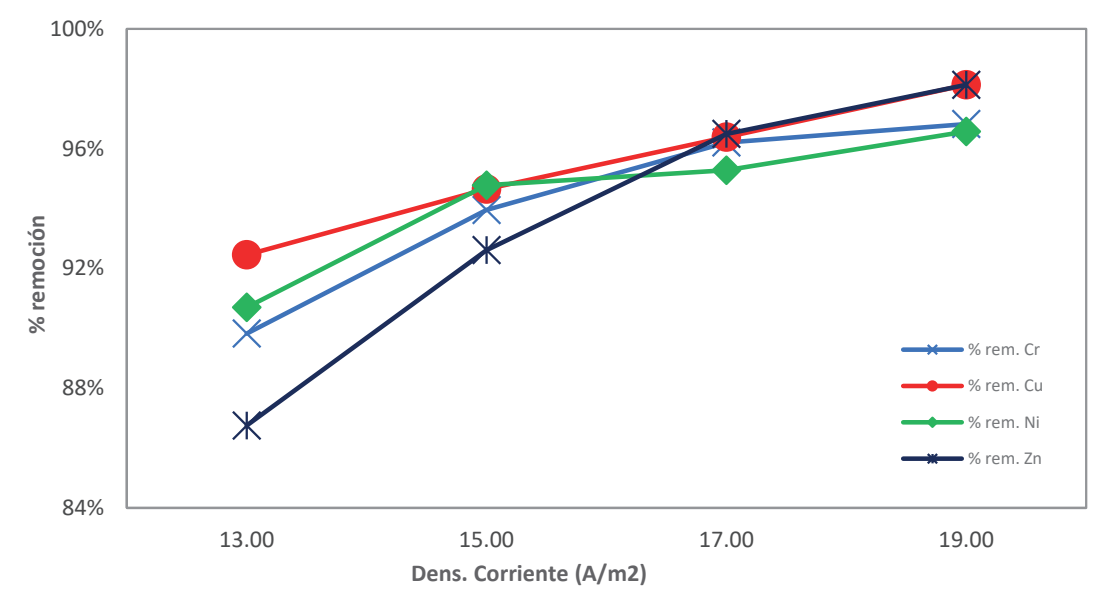

Figura 5. Efecto de la densidad de corriente $\left(\mathrm{A} / \mathrm{m}^{2}\right)$ sobre la eficiencia de remoción de metales pesados. Condiciones: $\mathrm{pH}$ 6, distancia entre electrodos $10 \mathrm{~mm}$, proceso continuo. Fuente: Elaboración propia 
residuales galvánicas, la cual se demostró su aplicabilidad en el tratamiento.

\section{AGRADECIMIENTOS}

El agradecimiento al Dr. Vidal Aramburu y a todas las personas e instituciones que de alguna manera me apoyaron a ser posible el desarrollo de la investigación. También a la Revista del Instituto de Investigación de la Facultad de Ingeniería Geológica, Minera, metalúrgica y Geográfica de la Universidad Nacional Mayor de San Marcos.

\section{REFERENCIAS}

Acosta Niño, G. E., Bourdón García, A., \& Coy Barrera, C. A. (2013). Evaluación y regulación de parámetros en un equipo diseñado para la disminución de Cromo(VI), provenientes de la industria de recubrimientos galvánicos. Ciencia e Ingeniería Neogranadina, 23(1), 107. https://doi org/10.18359/rcin.235

Adhoum, N., Monser, L., Bellakhal, N., \& Belgaied, J. E. (2004). Treatment of electroplating wastewater containing $\mathrm{Cu} 2+, \mathrm{Zn}$ $2+$ and $\mathrm{Cr}(\mathrm{VI})$ by electrocoagulation. Journal of Hazardous Materials, 112(3), 207-213. https://doi.org/10.1016/j. jhazmat.2004.04.018

Ardila Padilla, E. J. (2005). Evaluacion preliminar del proceso de electrólisis como alternativa para la recuperacion de metales pesados en la indrustria galvanica- estudio para níquel [Universidad de Los Andes]. https://repositorio. uniandes.edu.co/bitstream/handle/1992/10802/u263229. pdf? sequence $=1$

Chávez Porras, Á., Cristancho Montenegro, D., \& Ospina Granados, É. (2009). Una alternativa limpia para el tratamiento de las aguas residuales galvánicas: revisión bibliográfica. Revista Ingenierías Universidad de Medellín, $8(14)$, 39-50. https://revistas.udem.edu.co/index.php/ ingenierias/article/view/37

Chen, X., Chen, G., \& Lock Yue, P. (2000). Separation of pollutants from restaurant wastewater by electrocoagulation. Separation and Purification Technology, 19(9), 65 - $76 . \quad \mathrm{https} / / /$ citeseerx.ist.psu.edu/viewdoc/ download?doi=10.1.1.1072.2899\&rep=rep1\&type $=$ pdf

Franco Cogollo, J. E., \& Meza Cárcamo, D. J. (2017). Evaluación de la eficiencia de un reactor de electrocoagulación a escala piloto para la remoción de algunos parámetros fisicoquímicos y microbiológicos presentes en las aguas residuales. [Universidad Francisco de paula Santander Ocaña]. http://repositorio.ufpso.edu.co/xmlui/ handle/123456789/1678?locale-attribute $=$ en

Golder, A. K., Samanta, A. N., \& Ray, S. (2007). Removal of trivalent chromium by electrocoagulation. Separation and Purification Technology, 53(1), 33-41. https://doi. org/10.1016/j.seppur.2006.06.010

Holt, P. K., Barton, G. W., Wark, M., \& Mitchell, C. A (2002). A quantitative comparison between chemical dosing and electrocoagulation. Colloids and Surfaces A: Physicochemical and Engineering Aspects, 211(2-3), 233248. https://doi.org/10.1016/S0927-7757(02)00285-6
Jaramillo Páez, C. A., Jaramillo Giraldo, A., \& Taborda Ocampo, G. (2005). Construcción de un reactor electroquímico para electrocoagulación como método alternativo en la descontaminación de aguas residuales. Revista Universidad de Caldas, 145-158. https://www.academia.edu/30996900/ Construcción_de_un_reactor_electroquímico_para electrocoagulación_como_método_alternativo_en_la_ descontaminación_de_aguas_residuales

Khosla, N. K., Venkatachalam, S., \& Somasundaran, P. (1991). Pulsed electrogeneration of bubbles for electroflotation. Journal of Applied Electrochemistry, 21(11), 986-990. https://doi.org/10.1007/BF01077584

Kobya, M., Can, O. T., \& Bayramoglu, M. (2003). Treatment of textile wastewaters by electrocoagulation using iron and aluminum electrodes. Journal of Hazardous Materials, 100 , 163-178. https://doi.org/10.1016/S0304-3894(03)00102-X

Konstantinos, D., Achilleas, C., \& Evgenia, V. (2011). Removal of nickel, copper, zinc and chromium from synthetic and industrial wastewater by electrocoagulation. International Journal of Environmental Sciences, 1(5), 697-710. https:// www.semanticscholar.org/paper/Removal-of-nickel\%2Ccopper\%2C-zinc-and-chromium-from-Dermentzis-Christ oforidis/2af2720bb19e3379b3fb0609bc7ad08c1534a24f? p2df

Lavorante, M. J., Álvarez, H., Chantrill, C., \& Fasoli, H. (2011). Aplicaciones Ambientales de la Electrocoagulación a Escala de Planta Piloto Environmental Applications of Electrocoagulation at Pilot Plant Scale. Planta Piloto Ciencia Página, 6(39), 39. http://www.exactas.unca.edu.ar/ revista/v210/pdf/ciencia21-4.pdf

Mollah, M. Y. A., Morkovsky, P., Gomes, J. A. G., Kesmez, M., Parga, J., \& Cocke, D. L. (2004). Fundamentals, present and future perspectives of electrocoagulation. Journal of Hazardous Materials, 114(1-3), 199-210. https://doi. org/10.1016/j.jhazmat.2004.08.009

Nouri, J., Mahvi, A. H., \& Bazrafshan, E. (2010). Application of electrocoagulation process in removal of zinc and copper from aqueous solutions by aluminum electrodes. International Journal of Environmental Research, 4(2), 201-208. https://doi.org/10.22059/ijer.2010.10

Restrepo, P., Arango, Á., Ruiz, L., \& Garcés, F. (2006). La Electrocoagulación: retos y oportunidades en el tratamiento de aguas. Producción Mas Limpia, I, 20. https://www.researchgate.net/publication/277035194 La_Electrocoagulacion_retos_y_oportunidades_en_el_ tratamiento_de_aguas

Satish.I., C. (2013). Electrocoagulation: A Novel Waste Water Treatment Method. International Journal of Modern Engineering Research, 3(1), 93-100. http://www.ijmer.com/ papers/Vol3_Issue1/AP3193100.pdf

Vik, E. A., Carlson, D. A., Eikum, A. S., \& Gjessing, E. T. (1984). Electrocoagulation of potable water. Water Research, 18(11), 1355-1360. https://doi.org/10.1016/0043-1354(84)90003-4 